\title{
General anesthesia for a patient with multiple system atrophy
}

\author{
Myung-Soo Jang ${ }^{1,2}$, Jin Hee Han ${ }^{1,2}$, Sung Wook Park ${ }^{1,2}$, Jong-Man Kang ${ }^{1,3}$, and Wha Ja Kang ${ }^{1,2}$ \\ Department of Anesthesiology and Pain Medicine, ${ }^{1}$ Kyung Hee University College of Medicine, ${ }^{2}$ Kyung Hee University Hospital, \\ ${ }^{3}$ Kyung Hee University Hospital at Gangdong, Seoul, Korea
}

Multiple system atrophy (MSA) is a rare, adult-onset neurodegenerative disease [1]. Two clinical features characterize MSA: motor features and autonomic dysfunction [2]. During anesthesia, regulation of cardiovascular instability is an important issue. We report a case of successful general anesthesia in a MSA patient.

A 66-year-old man $(170 \mathrm{~cm}, 40 \mathrm{~kg})$ presented for a laparoscopic hemicolectomy. Recent intermittent hematochezia suggested rectal cancer. MSA was diagnosed at age 56, and rapid progression left him bedridden. His past medical history included diabetes and hypertension.

Preoperatively, no abnormal finding was detected on a laboratory examination, chest X-ray, or electrocardiography. In operating room, routine monitoring devices for electrocardiography, pulse oximetry, and non-invasive blood pressure, and a noninvasive cardiac output monitor showing cardiac output, cardiac index (CI), and stroke volume variation (SVV) were set up. To address possible cardiovascular instability, arterial cannulation was performed before anesthesia. A central venous catheter has already been inserted through the subclavian vein. Initial bispectral index (BIS) was 87 , blood pressure (BP) $150 / 60 \mathrm{mmHg}$, heart rate (HR) 75 beats/min, central venous pressure (CVP) 3 $\mathrm{cmH}_{2} \mathrm{O}, \mathrm{CI} 2.4 \mathrm{~L} / \mathrm{min} / \mathrm{m}^{2}$, and SVV $10 \%$.

General anesthesia was induced with etomidate $0.4 \mathrm{mg} / \mathrm{kg}$ intravenously. With good mask ventilation, $40 \mathrm{mg}$ of rocuronium was injected intravenously, and we tried laryngoscopic intubation. Because of poor mouth opening even after full relaxation with the rocuronium, moving the laryngoscope forward was impossible. So, we performed a bronchoscopic intubation. Anesthesia was maintained with sevoflurane $1.5-2$ vol\% and intravenous remifentanil $0.05 \mu \mathrm{g} / \mathrm{kg} / \mathrm{min}$ in an $\mathrm{O}_{2}$-air mixture at a $1: 1$ ratio. The operation lasted about $4 \mathrm{~h}$ and anesthesia was maintained appropriately with BIS at 35-50. At $25 \mathrm{~min}$ after induction of anesthesia, the BP abruptly fell from 160/85 to $80 / 50 \mathrm{mmHg}$. This was managed successfully with rapid fluid administration and a phenylephrine bolus dose of $40 \mu \mathrm{g}$ intravenously. HR hardly changed, from 82 to 90 beats/min. However, systolic blood pressure dropped again to $80 \mathrm{mmHg}$ during awakening despite the irritating sensation of the endotracheal tube. Phenylephrine $20 \mu \mathrm{g}$ raised the BP to $100 / 60 \mathrm{mmHg}$ again with little change in HR, 70-75 beats/min (Fig. 1). Finally, gentle extubation was tried, and no respiratory event occurred. He was transferred to the surgical intensive care unit, and has been cardiovascularly stable since then. He was returned to the ward next day.

The annual occurrence of MSA among those over 50 is $\sim 3$ per 100,000 with no gender predilection [1-3]. Currently, no definite diagnostic criteria or therapies exist, beyond supportive care [2].

There are several considerations regarding anesthesia in MSA. First, the anesthesiologist should be concerned about sudden hypotension due to dysautonomia. Several authors have reported that regional anesthesia is better than general anesthesia in maintaining cardiovascular stability in MSA because of absence of procedures that may aggravate hypotension, such as positive-pressure ventilation, and the avoidance of anesthetics for maintenance [3]. Nevertheless, there is no evidence that regional anesthesia is necessarily the right answer in MSA patients. In contrast, Cohen [1] reported a successful case of general anesthesia after failure of epidural anesthesia, induction

Corresponding author: Jin Hee Han, M.D., Ph.D., Department of Anesthesiology and Pain Medicine, Kyung Hee University Hospital, 23, Kyungheedae-ro, Dongdaemun-gu, Seoul 130-720, Korea. Tel: 82-2-958-8589, Fax: 82-2-958-8580, E-mail: esthesi@naver.com (c) This is an open-access article distributed under the terms of the Creative Commons Attribution Non-Commercial License (http:// creativecommons.org/licenses/by-nc/3.0/), which permits unrestricted non-commercial use, distribution, and reproduction in any medium, provided the original work is properly cited. 
A

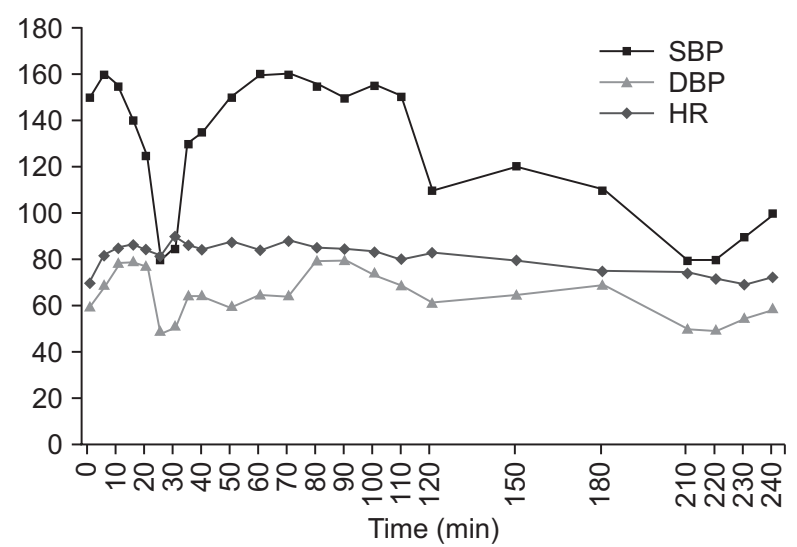

B

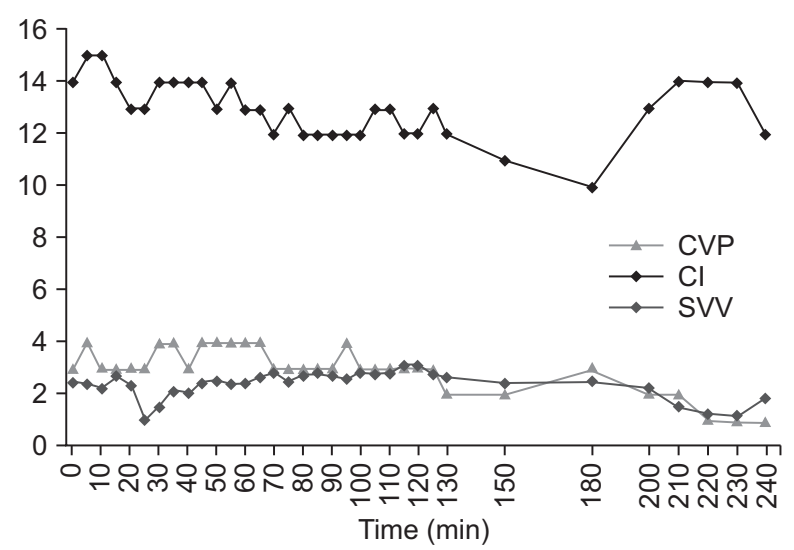

Fig. 1. Hemodynamic changes during anesthesia. (A) Indicates the changes in blood pressure and heart rate. There is an abrupt decrease in blood pressure to $80 / 50 \mathrm{mmHg}$ at $25 \mathrm{~min}$ after induction, but almost no change in the heart rate. Similarly, a sudden drop in blood pressure to $80 / 50 \mathrm{mmHg}$ occurs when there is a little change in heart rate, 70-75 beats/min; moreover, phenylephrine is effective in stabilizing the cardiovascular system. (B) Shows the fluctuation in CVP, CI, and SVV. There is a slight decrease in CVP and CI during the hypotension events with slight increases in SVV. SBP: systolic blood pressure $(\mathrm{mmHg}), \mathrm{DBP}$ : diastolic blood pressure $(\mathrm{mmHg}), \mathrm{HR}$ : heart rate (beats/min), CVP: central venous pressure $\left(\mathrm{cmH}_{2} \mathrm{O}\right), \mathrm{CI}$ : cardiac index $\left(\mathrm{L} / \mathrm{min} / \mathrm{m}^{2}\right)$, SVV: stroke volume variation $(\%)$.

with thiopental and succinylcholine, and maintenance with $\mathrm{N}_{2} \mathrm{O}$ and methoxyflurane. Ketamine was reported as a successful anesthetic in a MSA patient without hypotension in 1983 [4], because ketamine causes central sympathetic stimulation primarily through parasympathetic inhibition.

We decided on general anesthesia for several reasons: noncooperation, the possibility of procedural failure due to involuntary movements, and regional anesthesia not being suitable for laparoscopic surgery. The preoperative hemodynamic state of our patient was stable; however, realizing that MSA patients have a damaged sympathetic system, we decided to use etomidate with its minimal cardiovascular effects. We also used remifentanil, which has been reported to effectively suppress cardiovascular reactions and myoclonus after endotracheal intubation with etomidate [5].

Moreover, in MSA patients, bilateral vocal cord paralysis can be a life-threatening complication $[1,2]$. This adverse event can be aggravated during the induction or post-extubation period due to several stimuli, such as tracheal intubation under insufficient depth of anesthesia or unnecessary suction during extubation. If a preoperative evaluation is impossible, preventative measures should be used. Although no case has been reported of awake bronchoscopic intubation in MSA patients, it is worth considering in patients who are suspected to have difficult airways if the patients are cooperative. We also prepared an emergency cricothyroidotomy kit; the anesthesiologist should always be ready for an emergency tracheostomy situation.

In conclusion, anesthesia in MSA remains a great challenge due to the many potential complications. Anesthesiologists should consider the condition of patients with MSA and carefully decide on the appropriate method of anesthesia for the type of operation.

\section{References}

1. Cohen CA. Anesthetic management of a patient with the Shy-Drager syndrome. Anesthesiology 1971; 35: 95-7.

2. Wenning GK, Geser F, Krismer F, Seppi K, Duerr S, Boesch S, et al. The natural history of multiple system atrophy: a prospective European cohort study. Lancet Neurol 2013; 12: 264-74.

3. Malinovsky JM, Cozian A, Rivault O. Spinal anesthesia for transurethral prostatectomy in a patient with multiple system atrophy. Can J Anaesth 2003; 50: 962-3.

4. Saarnivaara L, Kautto UM, Teräväinen H. Ketamine anaesthesia for a patient with the Shy-Drager syndrome. Acta Anaesthesiol Scand 1983; 27: $123-5$.

5. Ko BJ, Oh JN, Lee JH, Choi SR, Lee SC, Chung CJ. Comparison of effects of fentanyl and remifentanil on hemodynamic response to endotracheal intubation and myoclonus in elderly patients with etomidate induction. Korean J Anesthesiol 2013; 64: 12-8. 